\title{
Efficacy of intravenous flurbiprofen axetil with epidural analgesia for post-cesarean section analgesia: a multi-center, prospective, randomized, blinded trial
}

\section{Ling-Yu Wang}

Guangzhou Women and Children Medical Center, Guangzhou Medical University

\section{Zu-Rong Hu}

Guangdong Women and Children Hospital

\section{Chun-Yuan Zhang}

Affiliated Bo-ai Hospital of Zhongshan, Southern Medical University

\section{Zai-Shen Dai}

He-Xian Memorial Hospital, Southern Medical University

\section{Bao-Yi Han}

Guangdong Women and Children Hospital

\section{Wei Zeng}

Affiliated Bo-ai Hospital of Zhongshan, Southern Medical University

\section{Hong-Tao Hu}

He-Xian Memorial Hospital, Southern Medical University

\section{Xing-Rong Song}

Guangzhou Women and Children Medical Center, Guangzhou Medical University

\section{Ying-Jun She ( $\nabla$ yjsheh@sohu.com )}

Guangzhou Women and Children Medical Center, Guangzhou Medical University

\section{Research Article}

Keywords: analgesia, cesarean section, flurbiprofen axetil, uterine contraction

Posted Date: August 24th, 2021

DOI: https://doi.org/10.21203/rs.3.rs-515788/v2

License: (c) (1) This work is licensed under a Creative Commons Attribution 4.0 International License. Read Full License 


\section{Abstract}

\section{Background}

Epidural analgesia provided satisfactory analgesic effects of incision pain after cesarean section. However, uterine contraction pain is also a common complication that cannot be relieved despite adequate levels of sensory blockade by epidural analgesia. This study evaluates the effect of flurbiprofen axetil with patient-controlled epidural analgesia on incision pain and uterine contraction pain after cesarean section.

Methods

A multi-center, prospective, randomized, blinded trial was performed. 1000 obstetric patients scheduled for cesarean delivery were randomized to receive epidural analgesia with intravenous flurbiprofen axetil (group EF) or placebo (group E) postoperatively. The primary outcomes were incision pain and uterine contraction pain score 48 hours after a cesarean section. Secondary outcomes were the PCEA attempts, the incidence of complication, satisfaction scores, and return of gastrointestinal (GI) function.

Results

Baseline characteristics were similar between groups. The VAS of uterine contraction pain was significantly lower in group EF than in group E from 12 hours to 48 hours after cesarean delivery $(\mathrm{P}<$ 0.01). The VAS of incision pain at rest and on movement was lower in group EF than in group $E$ from 12 hours to 48 hours after cesarean delivery. While sitting or walking, the VAS of incision pain was lower in group EF compared with that in group E. GI function recovered faster in group EF than that in group $\mathrm{E}$ (15 \pm 6 vs. $25 \pm 12,95 \% \mathrm{Cl} 5.7$ to $8.7, \mathrm{P}<0.01)$. The satisfaction scores are higher in group $\mathrm{EF}$ than that in group $\mathrm{E}(3.2 \pm 0.5$ vs. $2.9 \pm 0.5,95 \% \mathrm{Cl}-0.36$ to $-0.11, \mathrm{P}<0.01)$.

\section{Conclusion}

Combination flurbiprofen axetil with PCEA provides more effective analgesia for uterine contraction pain and incision pain after cesarean section, with a higher quality of recovery and better satisfaction scores.

Trials Registration

This study was approved by the Institutional Review Board of Guangzhou Women and Children's Medical Center (IRB2017062201) and written informed consent was obtained from his or her parents or legal guardians for each pediatric patient in the trial. The trial was registered before patient enrollment at chictr.org.cn (ChiCTR-IOR-17011956, Principal investigator: Ying-Jun She, Date of registration: 2017.07.12). Written informed consent was obtained from patients in the study.

\section{Background}


Pain is a common complication after cesarean section, including incision pain and uterine contraction pain. Unlike the stinging pain from the incision, uterine contraction pain is often referred to the visceral dull pain. Breastfeeding after cesarean section is known to stimulate the release of oxytocin, which can increase uterine tone and enhance uterine involution, resulting in intensive uterine contraction pain ${ }^{1}$. After a cesarean section, the anatomy of the uterus changes, the thinner lower segment, and the broken fiber cause weaker uterine contractility ${ }^{2}$. So it is common to give an oxytocin infusion to prevent postpartum hemorrhage, accompanied by severe uterine contraction pain ${ }^{1}$.

Post-cesarean section pain management is crucially important because obstetric patients must recover quickly to ambulate and care for their newborns ${ }^{3}$. A good analgesic plan allows early rehabilitation and helps prevent postpartum depression and chronic pain. However, Opioid-based analgesia during the postpartum period can disrupt breastfeeding, infant care, and lead to opioid-related adverse events ${ }^{4}$. Several studies indicated that patient-controlled epidural analgesia (PCEA) is helpful to relieve postpartum pain ${ }^{5}$, but the effects on uterine contraction pain have not been adequately investigated.

Limited studies exist that investigate the efficacy of intravenous drugs or epidural analgesia on uterine contraction pain and incision pain after cesarean section analgesia. Flurbiprofen axetil is a nonsteroidal anti-inflammatory drug (NSAID), with lipid microspheres as a carrier. It impairs the synthesis of prostaglandins by inhibiting COX-1 and COX-2, and it has a targeted analgesic effect ${ }^{6,7}$. Thus, the primary aim of this study was to evaluate the analgesic effect of flurbiprofen axetil with PCEA for incision pain and uterine contraction pain after cesarean section. We hypothesis that flurbiprofen axetil with PCEA relieves both uterine contraction pain and incision pain.

\section{Methods}

This study was approved by the Human Research Ethics Committee of Institutional Review Board of Guangzhou Women and Children's Medical Center (IRB2017062201). The trial was registered before patient enrollment at chictr.org.cn (ChiCTR-IOR-17011956, Principal investigator: Ying-Jun She, Date of registration: 2017.07.12). In the pre-op room, all eligible obstetric patients were informed about the study, and written informed patient consent was obtained for study participation.

The study was operated in four anesthesiology departments from August 2017 to December 2018. 1000 ASA physical status I or II obstetric patients scheduled for elective cesarean delivery at 8 am to noon were recruited into the study. Exclusion criteria included age younger than 18 years or older than 45 years, BMI $<18$ or $>35$, pregnancy-related hypertensive disease, cardiovascular, cerebrovascular or renal disease, bleeding disorders, infection at the site of injections, gestational age $<36$ weeks, drug abusers, allergic to NSAIDs, known abnormal fetal development, and other conditions that were considered unsuitable for this study by the attending anesthesiologists.

Using a computer-generated table, obstetric patients were randomly allocated into two groups (Epidural analgesia only group (group E); epidural analgesia plus intravenous flurbiprofen axetil group (group EF).) 
To ensure proper blinding, in each center there was one anesthetist responsible for the random allocation, performing the combined spinal-epidural anesthesia (CSEA), and managing the anesthesia procedure, the other anesthetist blinded to the group assignment was responsible for postoperative follow-up during surgery and analyzing the outcomes. All patients were unaware of group allocation.

All obstetric patients underwent preoperative fasting for 8 hours and water deprivation for 4 hours. After entering the operating room, standard monitors such as non-invasive blood pressure measurement, pulse oximetry, and electrocardiography were attached, the baseline was recorded. A 20-G IV catheter was placed in a peripheral vein in the obstetric patient's forearm, and a loading infusion of lactated Ringer's solution $10 \mathrm{ml} / \mathrm{kg}$ was commenced. In all patients, the skin was infiltrated with $1 \%$ Lidocaine, and CSEA was performed at the midline L3-4 interspace in the left lateral positions, $2.5 \mathrm{ml} 0.5 \%$ Ropivacaine was injected into the subarachnoid space in 10 seconds. After placing an epidural catheter, the patient was positioned at a $15^{\circ}$ left lateral tilt. Oxygen was administered through a face mask at a flow rate of $2 \mathrm{~L} / \mathrm{min}$ during the operation. The level of sensory block was evaluated by cold sensation and the level of motor block was recorded according to the modified Bromage scale ( $3=$ Cannot move foot or knee, $2=$ Can move foot only, 1 = Can flex the knee, move a foot, but cannot raise a leg, $0=$ no motor block). We adjusted the block level between T4 and T6 with an epidural supplement of $0.5 \%$ Ropivacaine. $0.25 \mathrm{mg}$ Palonosetron was given intravenously after delivery. At the end of the surgery, $0.1 \%$ Ropivacaine plus $2 \mathrm{mg}$ morphine in $8 \mathrm{ml}$ sterile saline was injected via an epidural catheter, then the catheter was connected with a PCEA pump. The PCEA pump was contained $200 \mathrm{ml} 0.1 \%$ Ropivacaine, the bolus was $3 \mathrm{ml}$, the lockout interval was 15 minutes, the infusion speed was $3 \mathrm{ml} / \mathrm{h}$. The patient in group EF was given $50 \mathrm{mg}$ flurbiprofen axetil intravenously after delivery, and then $50 \mathrm{mg}$ flurbiprofen axetil was administrated twice a day (7:00 and 19:00) intravenously until the third day after cesarean section. Group E was given saline as a placebo instead. Intravenous oxytocin was administrated during the surgery and from 7:30 to 8:00 the next morning.

We followed up patients 2 hours, 6 hours, 12 hours, 24 hours, and 48 hours after a cesarean section. The primary outcome was considered to be the VAS scale of the incision pain and uterine contraction pain. The secondary outcomes were the PCEA drug consumption and the quality of recovery. Side effects like dizziness, hypotension, palpitation, respiratory depression, pruritus, fever, postoperative nausea, and vomiting (PONV) were recorded. Adjuvant drugs, accumulated PCEA counts were recorded. Patients were asked to rank their satisfaction, according to the following scale: $1=$ unsatisfactory; $2=$ neutral; $3=$ satisfactory; and 4 = very satisfactory.

Statistical analyses

Sample size calculation was based on an initial pilot study where the standard deviation (SD) within each group was $3.1 \mathrm{~cm}$ VAS points 24 hours after a cesarean section. To achieve $90 \%$ power at $a=0.05$ level to detect a difference of $1 \mathrm{~cm}$ on the VAS between treatment and control groups, we needed a total of 774 patients (387 in each group). We recruited 1000 (500 in each group) patients to account for $15 \%$ of potential missing data or loss-to-follow up. 
Statistical analyses were performed in SPSS (IBM SPSS Statistics, Version 22, USA). The data were reported as mean (SD) and count (percentage) as appropriate and were analyzed using T-test or one-way ANOVA test. Qualitative variables were analyzed using chi-squared test. $\mathrm{P}<0.05$ were considered statistically significant.

\section{Results}

The CONSORT flow diagram shows the details of patient recruitment (Fig. 1.). One thousand patients were recruited into the study, 71 were excluded according to exclusion criteria, 455 were in group $E$ and 467 were in group EF. The groups were similar regarding maternal age, height, weight, BMl, gravida, parity, and gestational age $(P>0.05$, Table 1$)$.

The result of uterine contraction pain was shown in Fig. 2. The VAS of uterine contraction pain was significantly lower in group EF than that in group E from 12 hours to 48 hours after cesarean delivery $(P<$ 0.01). In addition, as the Fig. 3 shown, the VAS of incision pain at rest and on movement in group EF was lower than that in group $E$ from 12 hours to 48 hours $(P<0.01)$. From 24 hours to 48 hours after cesarean delivery, the VAS of incision pain when sitting or walking was lower in group EF compared with that in group $E(P<0.01)$.

The satisfaction scores are higher in group EF than that in group $\mathrm{E}(3.2 \pm 0.5 \mathrm{vs} .2 .9 \pm 0.5,95 \% \mathrm{Cl}-0.36$ to $-0.11, P<0.01)$. However, there was no significant difference in PCEA attempts between groups.

Results for side effects are shown in Table 2. Using the Pearson $\chi 2$ test, there was no significant difference in the incidence of nausea, vomiting, pruritus, dizziness, palpitation, and fever between groups. But the incidence of constipation in group $E$ was significantly higher than that in group $\operatorname{EF}(P=0.03)$. Also, the first exhaust time is shorter in group $\mathrm{EF}$, indicated that $\mathrm{Gl}$ function recovered faster in group $\mathrm{EF}$ than that in group $\mathrm{E}(15 \pm 6$ vs. $25 \pm 12,95 \% \mathrm{Cl} 5.7$ to $8.7, \mathrm{P}<0.01)$.

\section{Discussion}

An extensive literature search identified few studies with information regarding the incidence of uterine contraction pain after cesarean section. This multicenter randomized controlled trial investigated the effect of intravenous flurbiprofen axetil on postpartum uterine pain as part of a multimodal analgesic regimen inclusive of PCEA. Our study showed that combination with flurbiprofen axetil and PCEA provided better pain management of uterine contraction pain and incision pain to the patient.

Several studies ${ }^{8-10}$ concluded that PCEA is superior to PCIA in postoperative or labor analgesia management, with fewer adverse effects and greater patient satisfaction. In our study, PCEA provided satisfactory analgesic effects, the highest average VAS of incision pain at rest are 2.3 (in group E) and 1.6 (in group EF) respectively, which occurred 24 hours after surgery. During the cesarean section, patients often encountered uterine contraction pain despite adequate levels of sensory blockade. The uterus is innervated by sympathetic and parasympathetic nerves. The sympathetic nerve fibers of the 
uterus are from $T 5$ to $T 10$, so the blockade level is not enough to suppress the uterine contraction pain. A study ${ }^{7}$ concluded that visceral pain is transmitted by unmyelinated C-fibers. Although opioids such as fentanyl and morphine can depress C-fiber-mediated responses, sedation associated with opioids during the postpartum period may influence on breastfeeding and mother-infant bonding. Furthermore, the dosage of local anesthetic for postpartum analgesia is not enough to achieve T4 to T6 or depress Cfibers, thus it causes visceral pain. Therefore, an additional drug for visceral pain for improving uterine contraction pain is essential.

In our study, it showed that flurbiprofen axetil could release both uterine contraction pain and incision pain. Flurbiprofen axetil has a targeted analgesic effect for visceral pain. Hu et al. ${ }^{11}$ found out that the expression of $\mathrm{F}$ prostanoid receptors in postpartum myometrium was increased, the prostaglandin $\mathrm{F} 2$, oxytocin, acetylcholine-induced uterine contractions in rats. Nuray et al. ${ }^{12}$ pointed out that neutrophils, mast cells, and macrophages release inflammatory mediators like prostaglandins resulting in hyperalgesia in the injured and inflamed tissues. In vitro and in vivo studies indicated that flurbiprofen axetil can reduce pain by inhibiting the COX-2 resulting in the inhibition of prostaglandin synthesis ${ }^{13}$, which is considered as intrinsic myometrial stimulants, and is implicated in dysmenorrhea, the initiation of labor, and the postpartum uterine pain ${ }^{14}$. In addition, Flurbiprofen axetil can also enhance the analgesic effect for incision pain. Geng et al. ${ }^{15}$ concluded that flurbiprofen axetil was of high affinity in the incision of the patient undergoing tangential excision surgery. Besides, several studies pointed out that flurbiprofen axetil provided effective incisional analgesia, reduced opioid drug consumption and adverse effects for lower abdominal surgery ${ }^{6}$, total-knee arthroplasty ${ }^{16}$, gastrointestinal surgery ${ }^{17}$. Thus, not only visceral pain but also incision pain can be relieved by flurbiprofen axetil.

Our inclusion of morphine in the epidural analgesia may have slightly influenced the incidence of complications, particularly in the first 6 hours. Common side effects of morphine include nausea, vomiting, and pruritus. In the study, the rates of incidence of nausea, vomiting, and pruritus are $11 \%, 5 \%$, and $10 \%$ respectively. Kimura et al. ${ }^{18}$ demonstrated that flurbiprofen axetil improved nausea and emesis in 15 minutes after cesarean section. The mechanism might be the inhibition of cyclooxygenase. In our study, the incidence of nausea and vomiting are similar in both groups 2 days after a cesarean section. This discrepancy could be due to the use of palonosetron after delivery, which reduced the incidence of nausea and vomiting. According to large series, the incidence of pruritus of up to $5 \mathrm{mg}$ intrathecal morphine is less than $10 \%{ }^{19}$. Our study has shown that the incidence of pruritus in the flurbiprofen axetil group was decreased from $14-6 \%$, although with no statistical significance. Our results demonstrated an association between intravenous flurbiprofen axetil and shorter gastrointestinal recovery time. It's possibly because flurbiprofen axetil relieved uterine contraction pain, made the mothers more willing to move, enhanced gastrointestinal movement, and helped the mother performed their daily activities normally.

There are two limitations in this study. First, the time of oxytocin and flurbiprofen administration would affect the postoperative pain score at different periods. Oxytocin would be administrated routinely at 7:30 
am on the first day after cesarean section, and flurbiprofen axetil was administrated at 7:00 and 19:00 for postoperative two days. However, the cesarean section was finished at different times, and the postoperative pain scores would be affected. Therefore we recruited obstetric patients scheduled for elective cesarean delivery from 8 am to noon to mitigate this influence. Secondly, we don't have a dosedependent study, so we should further determine the optimal dose.

\section{Conclusions}

In conclusion, combination flurbiprofen axetil with PCEA provides more effective analgesia for uterine contraction pain and incision pain after cesarean section, with a higher quality of recovery and better satisfaction scores.

\section{List Of Abbreviations}

patient-controlled epidural analgesia (PCEA)

patient-controlled intravenous analgesia (PCIA)

nonsteroidal anti-inflammatory drug (NSAID)

combined spinal-epidural anesthesia (CSEA)

postoperative nausea and vomiting (PONV)

gastrointestinal (GI)

visual analgesia scale (VAS )

\section{Declarations}

\section{Ethics approval and consent to participate}

This study was approved by the Human Research Ethics Committee of Institutional Review Board of Guangzhou Women and Children's Medical Center (IRB2017062201). The trial was registered before patient enrollment at chictr.org.cn (ChiCTR-IOR-17011956, Principal investigator: Ying-Jun She, Date of registration: 2017.07.12). Informed consent was obtained from of all individual participants included in the study.

\section{Consent for publication}

Not applicable

\section{Availability of data and materials}


The datasets used and analyzed during the current study are available from the corresponding author on reasonable request.

\section{Competing interests}

The authors declare that they have no competing interests.

\section{Funding}

This study was supported by department funding of Guangzhou Women and Children Medical Center.

\section{Authors' contributions}

XR.S and YJ.S designed and conducted the study. LY.W collected, analyzed, and interpreted the data; and wrote the manuscript. Z.H, CY.Z, Z.D were helped design and conduct the study. BH, WZ, HTH were participated in collecting and interpreting the data. All authors read and approved the final manuscript.

\section{Acknowledgements}

Not applicable

\section{Authors' information}

Dr.Ying-Jun She, Department of Anesthesiology and Perioperative Medicine, Guangzhou Women and Children Medical Center, Guangzhou Medical University. 9 Jinsui Road, Tianhe District, Guangzhou 510000, China. Email: yjsheh@sohu.com

\section{References}

1. Handlin L, Jonas W, Petersson M, Ejdeback M, Ransjo-Arvidson AB, Nissen E, Uvnas-Moberg K (2009) Effects of sucking and skin-to-skin contact on maternal ACTH and cortisol levels during the second day postpartum-influence of epidural analgesia and oxytocin in the perinatal period. Breastfeed Med 4 (4):207-220. doi:10.1089/bfm.2009.0001

2. Holdcroft A, Snidvongs S, Cason A, Dore CJ, Berkley KJ (2003) Pain and uterine contractions during breast feeding in the immediate post-partum period increase with parity. Pain 104 (3):589-596

3. Chi X, Li M, Mei W, Liao M (2017) Comparison of patient-controlled intravenous analgesia with sufentanil versus tramadol in post-cesare an section pain management and lactation after general anesthesia - a prospective, randomized, doubl e-blind, controlled study. J Pain Res 10:1521-1527

4. Urman RD, Boing EA, Pham AT, Khangulov V, Fain R, Nathanson BH, Zhang X, Wan GJ, Lovelace B, Cirillo J (2018) Improved Outcomes Associated With the Use of Intravenous Acetaminophen for Management of Acute Post-Surgical Pain in Cesarean Sections and Hysterectomies. Journal of clinical medicine research 10 (6):499-507. doi:10.14740/jocmr3380w 
5. Lalmand M, Wilwerth M, Fils JF, Van der Linden P (2017) Continuous Ropivacaine Subfascial Wound Infusion Compared With Intrathecal Morphine for Postcesarean Analgesia: A Prospective, Randomized Controlled, Double-Blind Study. Anesth Analg 125 (3):907-912

6. Xiang X, Yuan X, Lian Y, Fang J, Wu Y (2018) Effect of oxycodone hydrochloride combined with flurbiprofen axetil for intravenous patient-controlled analgesia in lower abdominal patients: A randomized trial. Medicine (Baltimore) 97 (7):e9911. doi:10.1097/MD.0000000000009911

7. Ishiyama T, Yamaguchi T, Kashimoto S, Kumazawa T (2001) Effects of epidural fentanyl and intravenous flurbiprofen for visceral pain during cesarean section under spinal anesthesia. J Anesth 15 (2):69-73. doi:10.1007/s005400170029

8. Halpern SH, Muir H, Breen TW, Campbell DC, Barrett J, Liston R, Blanchard JW (2004) A multicenter randomized controlled trial comparing patient-controlled epidural with intravenous analgesia for pain relief in labor. Anesth Analg 99 (5):1532-1538; table of contents.

doi:10.1213/01.ANE.0000136850.08972.07

9. Hwang BY, Kwon JY, Jeon SE, Kim ES, Kim HJ, Lee HJ, An J (2018) Comparison of patient-controlled epidural analgesia with patient-controlled intravenous analgesia for laparoscopic radical prostatectomy. Korean J Pain 31 (3):191-198. doi:10.3344/kjp.2018.31.3.191

10. Kampe S, Randebrock G, Kiencke P, Hunseler U, Cranfield K, Konig DP, Diefenbach C (2001) Comparison of continuous epidural infusion of Ropivacaine and sufentanil with intravenous patientcontrolled analgesia after total hip replacement. Anaesthesia 56 (12):1189-1193

11. Hu C, Liu B, Li H, Wu X, Guo T, Luo W, Zhou Y (2018) Prostaglandin D2 evokes potent uterine contraction via the $F$ prostanoid receptor in postpartum rats. Eur J Pharmacol 836:11-17

12. Bozkurt N, Kurdoglu M, Kurdoglu Z, Kutlusoy F, Biberoglu K (2009) Postoperative pain control after cesarean section: can diclofenac sodium be used instead of meperidin e? J Matern Fetal Neonatal Med 22 (12):1144-1150

13. Graham DJ (2006) COX-2 inhibitors, other NSAIDs, and cardiovascular risk: the seduction of common sense. Jama 296 (13):1653-1656

14. Bloomfield SS, Mitchell J, Cissell G, Barden TP (1986) Flurbiprofen, aspirin, codeine, and placebo for postpartum uterine pain. Am J Med 80 (3A):65-70

15. Geng W, Hong W, Wang J, Dai Q, Mo Y, Shi K, Sun J, Qin J, Li M, Tang H (2015) Flurbiprofen Axetil Enhances Analgesic Effects of Sufentanil and Attenuates Postoperative Emergence A gitation and Systemic Proinflammation in Patients Undergoing Tangential Excision Surgery. Mediators Inflamm 2015:601083

16. Xiao X, Zhang Q, Ouyang Z, Guo X (2018) Comparison of perioperative flurbiprofen axetil or celecoxib administration for pain management after total-knee arthroplasty: A retrospective study. Medicine (Baltimore) 97 (37):e12391. doi:10.1097/MD.0000000000012391

17. Mao Y, Cao Y, Mei B, Chen L, Liu X, Zhang Z, Gu E (2018) Efficacy of Nalbuphine with Flurbiprofen on Multimodal Analgesia with Transverse Abdominis Plane Block in Elderly Patients Undergoing Open 
Gastrointestinal Surgery: A Randomized, Controlled, Double-Blinded Trial. Pain Res Manag 2018:3637013. doi:10.1155/2018/3637013

18. Kimura M, Okamoto T, Tsukagoshi H, Sato J, Saito S (2011) Effect of flurbiprofen, metoclopramide and droperidol for nausea and emesis during cesarean section under spinal anesthesia. Journal of Anesthesia 25 (5):692-697. doi:10.1007/s00540-011-1203-7

19. Kung C-C, Chen S-S, Yang H-J, Lai C-J, Chen L-K (2018) Pharmacogenetic study of pruritus induced by epidural morphine for post cesarean section analgesia. Taiwanese Journal of Obstetrics and Gynecology 57 (1):89-94. doi:10.1016/j.tjog.2017.12.015

\section{Tables}

Please see the supplementary files section to view the tables.

\section{Figures}




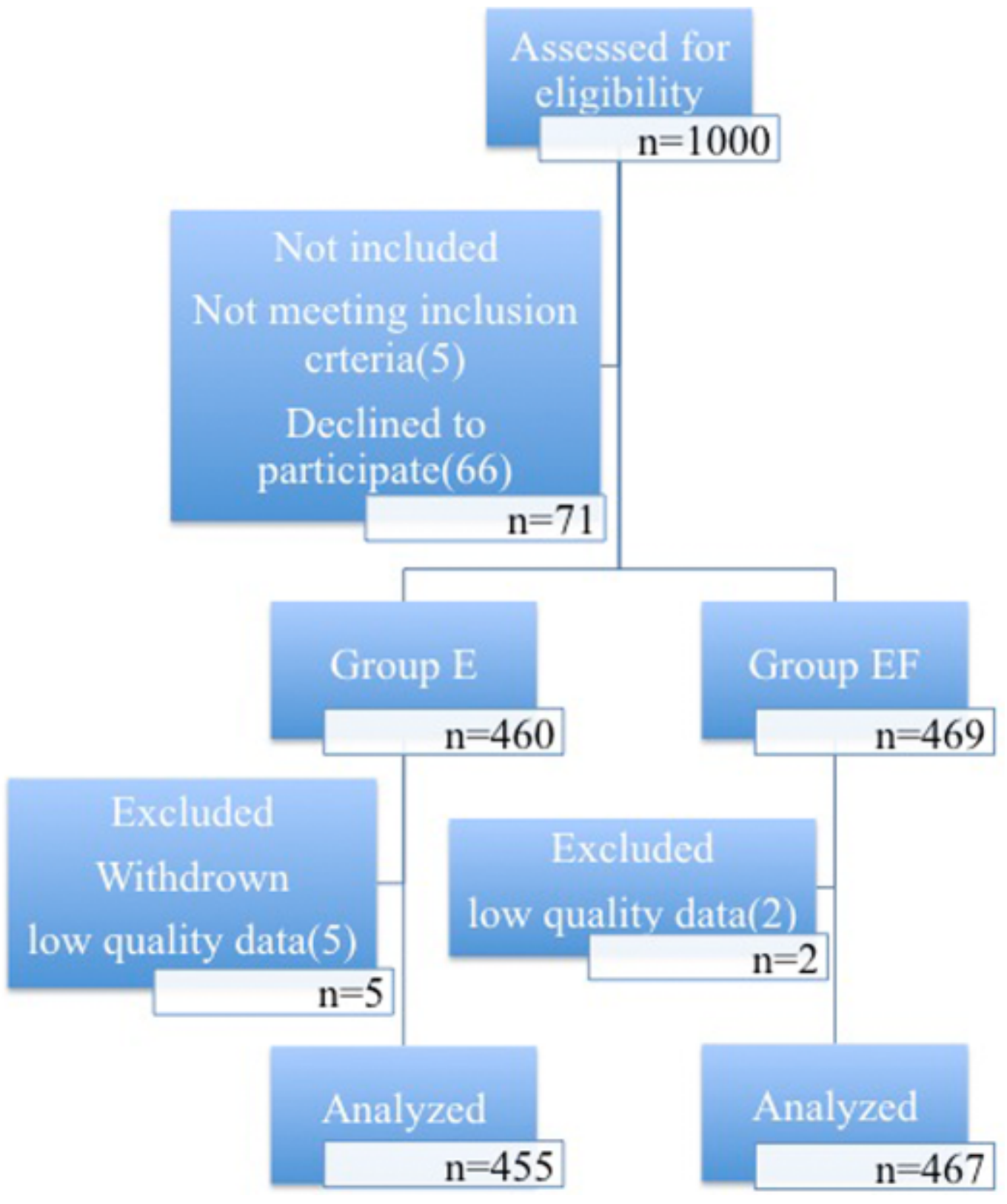

Figure 1

CONSORT flow diagram. 


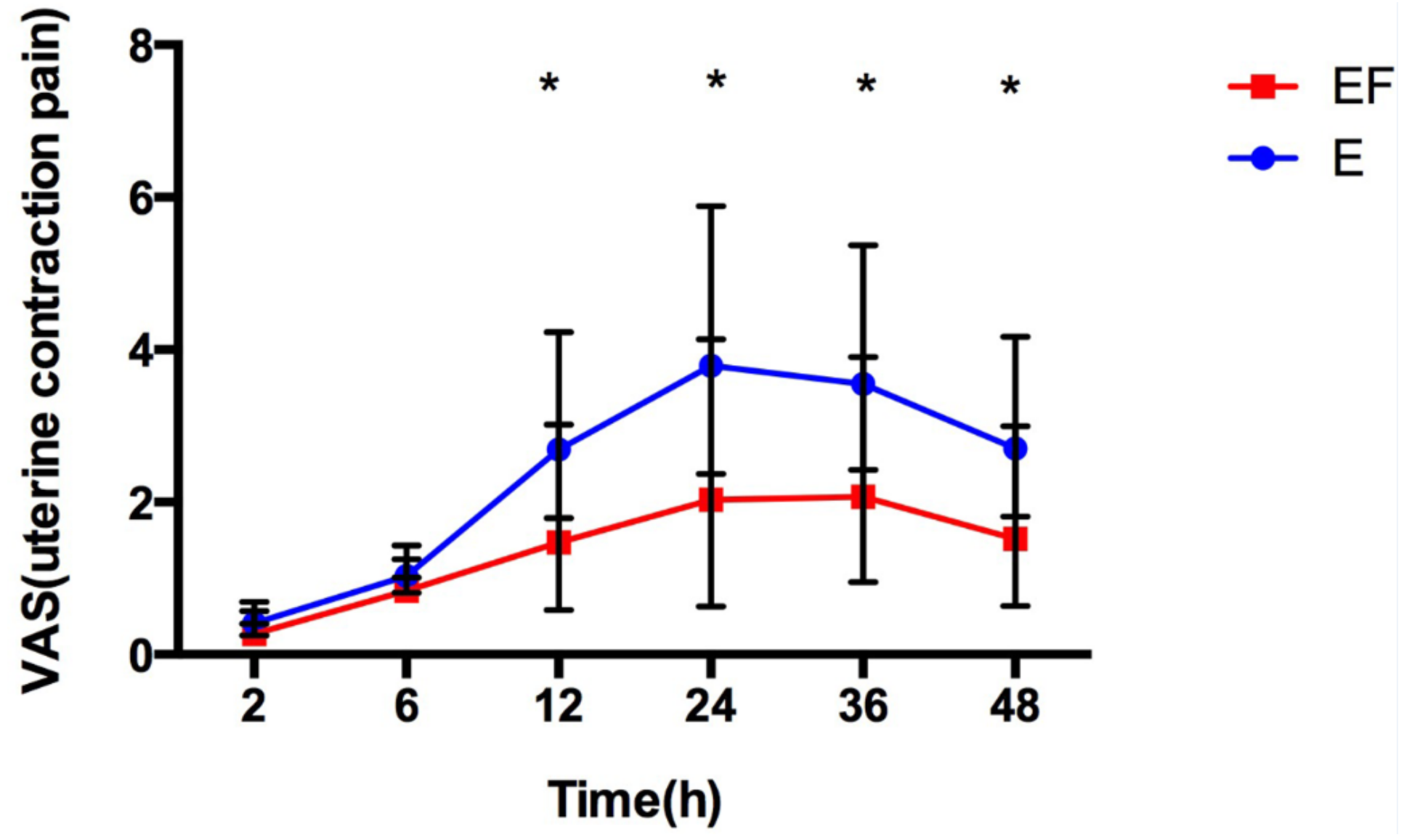

Figure 2

Uterine contraction pain intensity (VAS, 0-10), presented as mean $(95 \% \mathrm{Cl})$. VAS, visual analogue scale. * $\mathrm{P}<0.05$. 

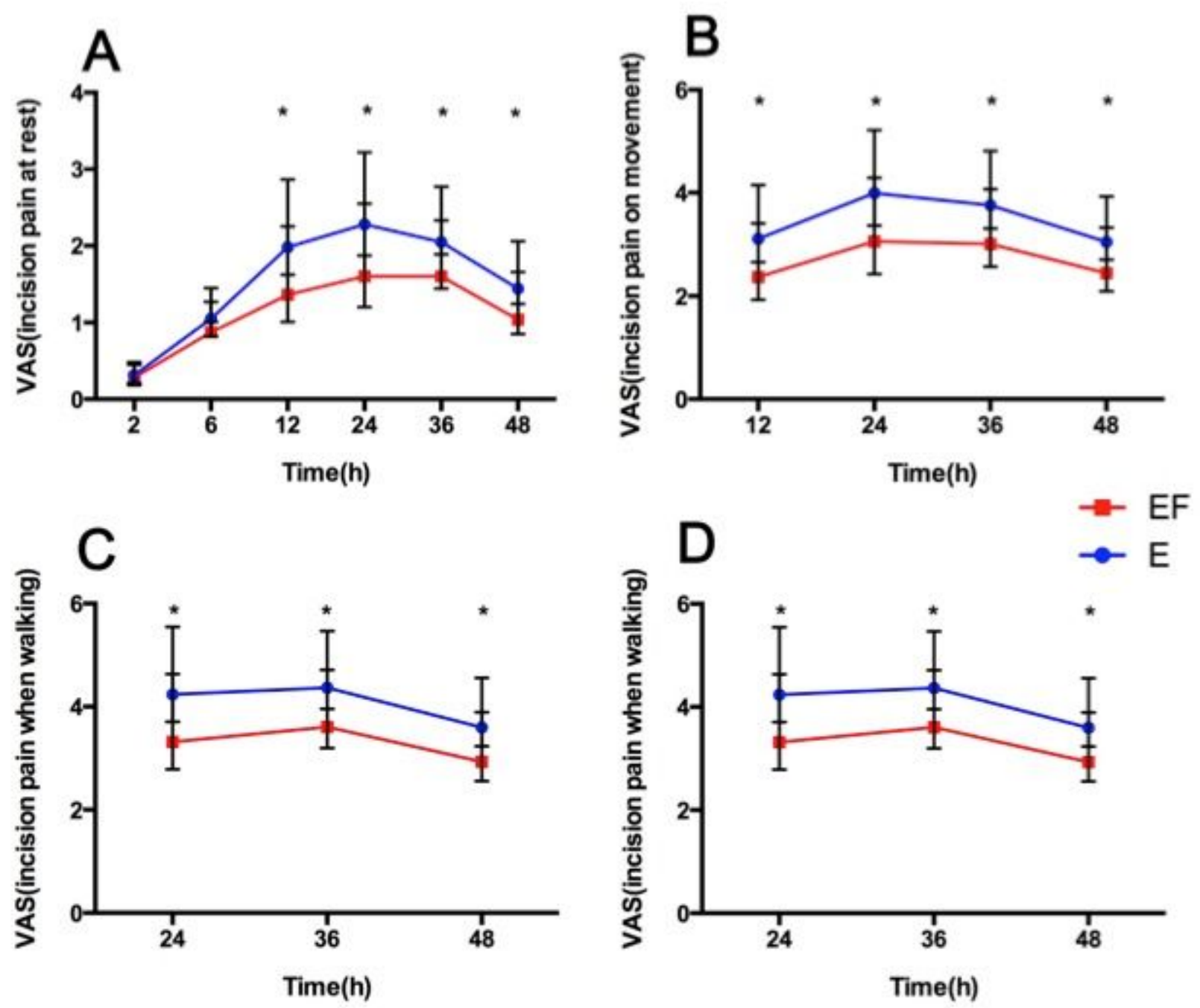

Figure 3

Pain intensity (VAS, 0-10) at rest (A), movement in bed (B), when sitting (C) and walking (D), presented as mean $(95 \% \mathrm{Cl})$. VAS, visual analogue scale. *, $\mathrm{P}<0.05$.

\section{Supplementary Files}

This is a list of supplementary files associated with this preprint. Click to download.

- Table.pdf 